\title{
Research on Network Scalability Based on UWB Indoor Localization System
}

\author{
Chunhua Long, Chong Shen, Gaoang Feng, Yuhao Zhu and Zhihao Wang \\ Hainan University, Haikou and 570228, China
}

\begin{abstract}
Network Scalability is an important step in the development of UWB indoor localization system. Owing to its fine resolution and robustness in cluttered environments, UWB signals are well suited for localization purposes as they allow high ranging accuracy. TDOA algorithm based on UWB indoor localization system can achieve highprecision positioning but it requires each anchor clock synchronization. This paper mainly studied the optional of master anchor based on TDOA algorithm. It becomes an effective way to adapt to the complex communication environment. Then realized the network scalability in multiple anchor conditions and finally evaluated the performance of master anchor optional algorithm on a realistic indoor scenario. A localization error analysis on UWB indoor positioning system was performed. The smallest TDOA measurement error is $15.127 \mathrm{~cm}$ and the worst error is $27.230 \mathrm{~cm}$, as was shown by the test results.
\end{abstract}

\section{Introduction}

Indoor localization has been an area of intensive investigation since it has great demands in many applications, such as localization-based billing in store or market, tools and equipment positioning in factory [1]. Localization accuracy is highly dependent on the signal parameters and especially on the wireless technology used since it determines the quality of the estimation of those parameters.

There are several different radio technologies such as WiFi, Bluetooth, Zig Bee or Ultra-wideband (UWB) with different levels of accuracy, range and complexity used for indoor localization system and localization systems based on RSSI estimation are not very suitable for indoor location because RSSI is very sensitive to multipath and non-line-of sight (NLOS) situations and their accuracy is usually within a few meters [2]. In this wireless positioning technologies, there is nothing comparable to UWB (Ultra wideband) wireless positioning technology to achieve a series of requirements of future wireless positioning. UWB technology is well suited for communication in wireless sensor networks, in which low power consumption for data transmission and the availability of precise ranging information are highly desirable [3]. With a centimeter-level ranging resolution, UWB is one of the most promising technologies to provide indoor location [2]. Moreover, the positioning accuracy using UWB is higher and can reach $10-20 \mathrm{~cm}$.

At present, there are many start-up companies joined the research for UWB high precision positioning technology. DecaWave is a very good example. They not only successfully developed the positioning technology of UWB chip, but also released solutions to 3D positioning accuracy based on different occasions. DecaWave released a wireless indoor positioning chip which called ScenSor. It has high accuracy and low energy consumption characteristics and its first product model DW 1000 have a minimum error within $10 \mathrm{~cm}$.

In daily communication, UWB with its special advantages can realize high quality and high transmission data, but the power of UWB seriously affects the transmission distance. Therefore, the use of UWB for signal transmission is more suitable for personal area network. Although the existing positioning algorithm is comparatively mature, but in many field environment, it is not good to use UWB technology to achieve precise positioning and it's hard to carry out. For example: TOA [4] (Time of Arrival) algorithm for positioning should keep the anchor and the mobile tag absolute clock synchronization. TOF (Time of Flight) [5] location method has a key constraints the sender and the receiver require high precision timer. TDOA (Time Difference of Arrival) requires location between anchors to keep strict clock synchronized and positioning will be affected if two regions of the clock are out of synchronization. But a system only has one master anchor and when the master anchor broken, the reliability of the system will be degraded. So, how to resolve these two problems becomes the target of this research.

In this work, details of the theory on TDOA algorithm is given, followed by an error analysis mainly on propagation and clock drifts. A $500 \mathrm{MHz}$ experimental 
UWB localization system was built. The s mallest TDOA measurement error is around $15 \mathrm{~cm}$ and the worst error is around $27 \mathrm{~cm}$, as was shown by the test results.

The paper is organized as follows. In Section 2, we present the TDOA algorithm. In Section 3, we describe the principle of network scalability in UWB indoor localization system and put forward the Master anchor optional algorithm. In section 4, we give the experiment environment and analyze the experiment results. Finally, we draw some conclusions.

\section{UWB Indoor Localization System Based On TDOA Algorithm}

In UWB indoor positioning system, due to UWB signal has very wide bandwidth and high time resolution, the technology based on TDOA can realize very accurate positioning. In [6], it came to conclusion that for the case of all NLOS channels, we derive a simple condition among the number of cells, the average and variance of NLOS range error distribution which can correctly predict whether the TDOA method outperforms the TOA method or not.

In our experiment, the algorith $\mathrm{m}$ based on TDOA [7] is used.

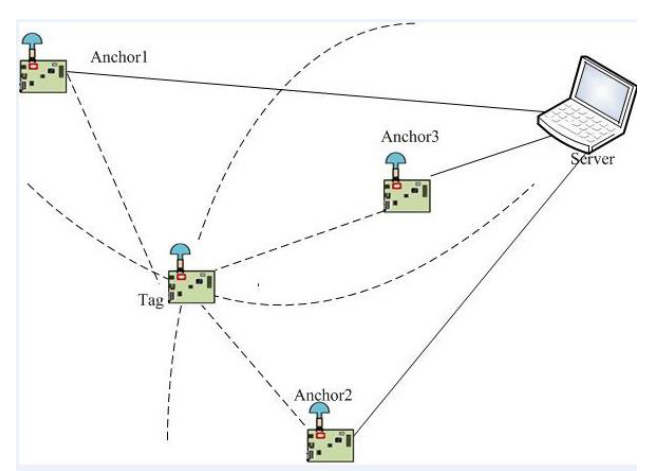

Figure1. TDOA positioning model

The principle of TDOA is similar to TOA. In Figure 1, the dotted line represents the wireless signal. Anchor 1, 2 and 3 receive a radio signal sent by mobile tag and record the time of $T_{A 1}, T_{A 2}$ and $T_{A 3}$, and the data will be transferred to the data analysis center. Assume that tag receives the signal from anchor 1 at $T_{A 1}$ and receives the signal from anchor 2 at $T_{A 2}$. The signal is transmitted by anchor 1 and 2 at $T_{1}$ and $T_{2}$ respectively. $\Delta T_{1}, \Delta T_{2}$ and $\Delta T_{A}$ are the time difference between the time reference of anchor 1, 2 and tag with the time standard reference respectively.

If $T O A_{1}$ is the arrival time from anchor 1 to the tag [8]:

$$
T O A_{1}=\left(T_{A 1}+\Delta T_{A}\right)-\left(T_{1}+\Delta T_{1}\right)
$$

And $\mathrm{TOA}_{2}$ is the arrival time from anchor 2 to the tag:

$$
T O A_{2}=\left(T_{A 2}+\Delta T_{A}\right)-\left(T_{2}+\Delta T_{2}\right)
$$

Then, the TDOA between anchor 1 and 2 equals:

$$
\begin{aligned}
& \text { TDOA } \\
& 2,1 \\
& \left.=T_{A 2}+\Delta T_{2}-T_{A}\right)-\left(T_{2}+\Delta T_{2}\right)-\left(T_{A 1}+\Delta T_{A}\right)-\left(T_{1}+\Delta T_{1}\right) \\
& =\left(\mathrm{T}_{A 2}-T_{A 1}\right)-\left(T_{2}-T_{1}\right)+\left(\Delta T_{1}-\Delta T_{2}\right)
\end{aligned}
$$

Due to the time division style is adapted, the time difference of sending between two anchors is a fixed constant $T^{\prime}$, that is $T_{2}-T_{1}=T^{\prime}$.What is more, TDOA mode decides that the clock of each anchor is synchronous, so $\Delta T_{1}-\Delta T_{2}=0$, then

$$
T D O A_{2,1}=T O A_{2}-T O A_{1}=\left(T_{A 2}-T_{A 1}\right)-T^{\prime}
$$

As long as $T_{A 2}$ and $T_{A 1}$ are known, the TDOA value between anchor1 and anchor2 can be required. At the same time, the TDOA values between other anchors can be required with the same method.

$$
\begin{aligned}
& c \times T D O A_{1,2} \\
& =\sqrt{\left(x-x_{2}\right)^{2}+\left(y-y_{2}\right)^{2}+\left(z-z_{2}\right)^{2}} \\
& -\sqrt{\left(x-x_{1}\right)^{2}+\left(y-y_{1}\right)^{2}+\left(z-z_{1}\right)^{2}} \\
& c \times T D O A_{2,3} \\
& =\sqrt{\left(x-x_{2}\right)^{2}+\left(y-y_{2}\right)^{2}+\left(z-z_{2}\right)^{2}} \\
& -\sqrt{\left(x-x_{3}\right)^{2}+\left(y-y_{3}\right)^{2}+\left(z-z_{3}\right)^{2}} \\
& c \times T D O A_{1,3} \\
& =\sqrt{\left(x-x_{1}\right)^{2}+\left(y-y_{1}\right)^{2}+\left(z-z_{1}\right)^{2}} \\
& -\sqrt{\left(x-x_{3}\right)^{2}+\left(y-y_{3}\right)^{2}+\left(z-z_{3}\right)^{2}}
\end{aligned}
$$

Then, the location coordinate $(x, y, z)$ of the tag can be solved according to the group of equation (5). $\left(x_{1}, y_{1}, z_{1}\right) 、\left(x_{2}, y_{2}, z_{2}\right)$ and $\left(x_{3}, y_{3}, z_{3}\right)$ are the location coordinate of anchor 1 , anchor 2 and anchor3.

TDOA method has the advantages that when calculating the TDOA value, the calculation error is the same and zero for all base stations, these errors include public multipath delay and synchronization error.

\section{Network Scalability based on UWB Indoor Localization system}

\subsection{The Principle of Network Scalability}

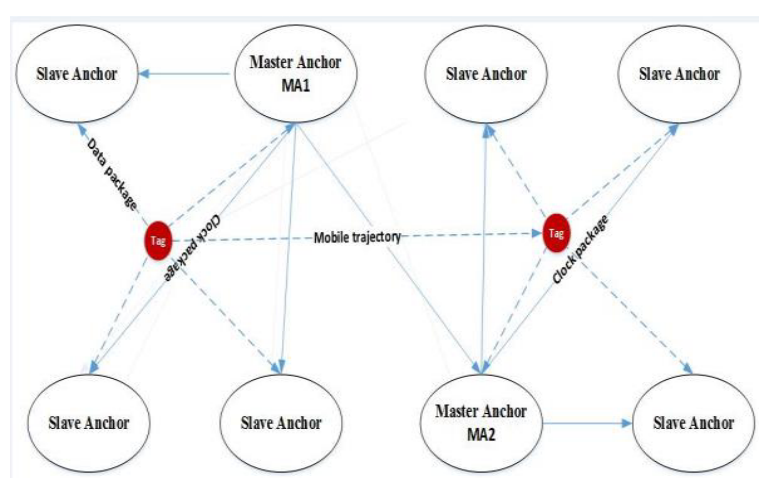

Figure2. LAN of master-slave clock synchronization 
Network scalability is the key research content of realizing large capacity multi-label real-time accurate positioning. The premise of network scalability is to ensure clock synchronization between the various regional networks or in s large room. However, the difference between LAN (Local Area Network) environments is difficult to achieve clock synchronization. This paper studies the relative clock synchronization of master anchors in different regions network. Namely, all the anchors in the area have been relative clock synchronization. The principle diagram was shown in Figure 2.

We set two master anchors at first and they are responsible for sending the clock synchronization information to the corresponding slave anchors. The clock signal sent by the master anchor MA1, clock synchronization information from the MA1 will be received by the slave anchors and the slave anchors adjust its clock signal to ensure network clock synchronization. Show in Figure 2, when the indoor area is large, the anchor MA2 in the intersection of the two areas of the network will sent the clock synchronization in formation which receives from MA1 separately to the adjacent area network. Each anchor has its own ID. It will have a delay, but this does not affect the real-time precise positioning of tags in the respective area network. From the above principle: relative clock synchronization of multiple LAN ensures network scalability to achieve.

In this UWB indoor localization system we will use the Kalman filter algorithm to solve the problem of time out-of-synchronization. The filter handles incomplete and noisy measurement-data by minimizing the mean squared error.

The advantage of the above method is that can achieve a wide range of regional relative clock synchronization. So it can achieve precise positioning and good scalability. The disadvantage is the master anchor greatly influences the reliability and stability of the performance of the whole positioning system.

\subsection{Master Anchor Optional Algorithm}

The key technology of network scalability is to reduce the comple xity of the adaptive a lgorith $m$ and the trans mission power of the anchor. In TDOA algorithm, the slave anchors only receive clock synchronization information from master anchor. The master anchor needs not only to send the clock synchronization information to the slave anchor, but also needs to maintain communication with the tag. So the master anchor adaptive selection determines the accuracy of the whole regional network location to a great extent.

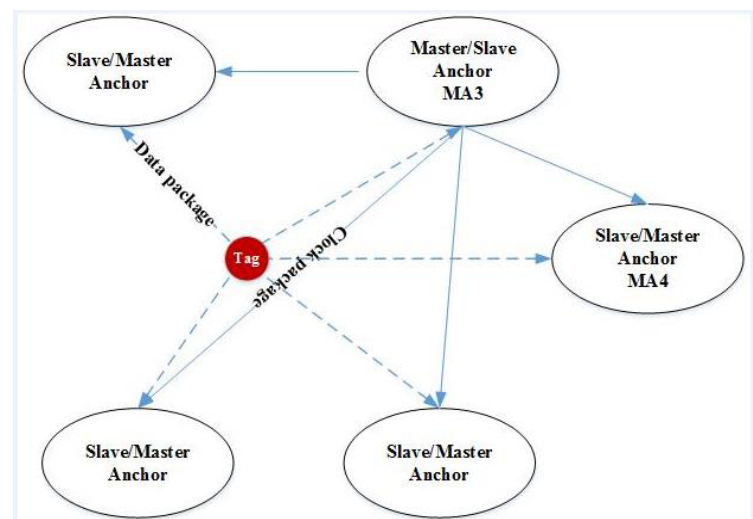

Figure3. Master anchor optional method

As shown in Figure 3, we put forward a Master anchor optional algorithm. All anchors in the regional network are set with two modes: the master anchor and slave anchor mode. When the master anchor MA3 broken, the next slave anchor MA4 can automatically become the primary master anchor, which can ensure the continuation of the implementation of the whole network system.

This method only increases the number of anchors. Figure 4 is a master anchor optional algorith $\mathrm{m}$ flow chart. Tag sends data package to the all anchors. If it found that it can't communication with the master anchor. It will automatically select next master anchor to transmit clock synchronization information to the slave anchors.

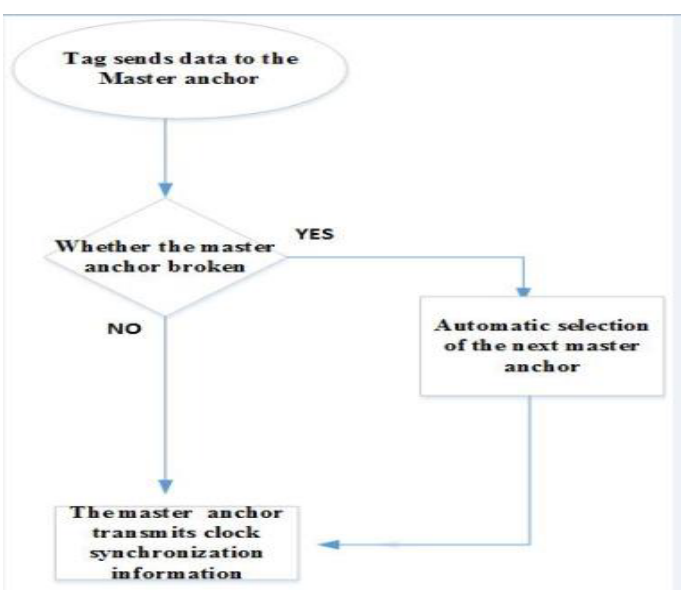

Figure4. Master anchor optional algorithm flow chart

\subsection{Selection of anchor location}

Due to the receiver noise in each anchor and errors associated with multipath and signal shadowing effects, delay of clock, there will be errors in the measurement of localization. GDOP provide guidance to the design of short to medium-range outdoor and indoor locating systems [9]. At the same time, [9] put forward the analysis of GDOP is an essential feature in determining the performance of a positioning system. At the same time, [12] put forward that the accuracy of a position estimate can be expressed as the product of the GDOP at a point and the root-mean-square error (RMSE) of the ranging errors from anchors to the moving tag. When the time error is definite, the GDOP value is higher resulting in 
poorer positioning performance while the GDOP value is lower resulting in higher positioning performance.

So, when we set up the anchor and the selection of the master anchor, we will take this factor into account.

And the algorithm priority selects the master anchor with high coordinate. Because the higher the coordinates, the higher the probability of the obstruction, the higher the accuracy of the positioning.

\section{Experiment AND results}

We used TDOA positioning algorithm for mobile tag, mobile tag and anchors were unobstructed. As [10] mentioned, in the condition of NLOS, no localization information would be got without priori channel knowledge, thus we only tested in the LOS [11] (line of sight) environment.

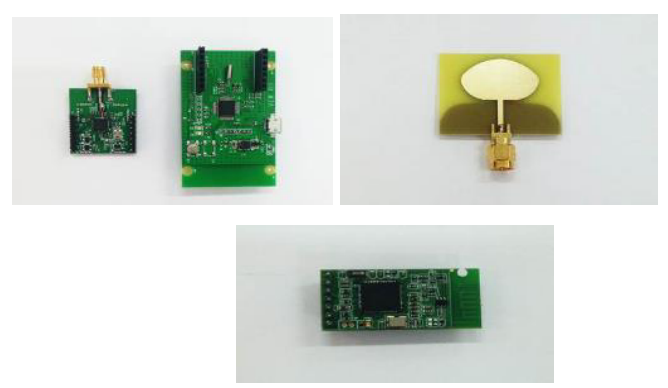

Figure 5. Anchor

Figure 5 is the DW1000, UWB antenna and Wi-Fi module. It can estimate the resultant distance between the two boards and display that result on the on-board display. Only external powering is required for this operation. It has an equivalent PC application which can be connected to the anchor via the mic ro USB, to control the DW 1000 from a PC.

Consider a localization system defined by the geometry of a mobile tag and anchors. The true position of the mobile tag is at the origin of the coordinate system. And in our experiment, we used localization error to measure the performance of the positioning system which is shown in Figure 7 and Figure 9. In this study, we used only one mobile label. Localization error is defined as follows:

$$
\mathrm{e}_{L}=\sqrt{(x-\bar{x})^{2}+(y-\bar{y})^{2}}
$$

Where $\mathrm{e}_{L}$ is the localization error, $(\bar{x}, \bar{y})$ is the Tag's measurement coordinate location and $(x, y)$ is Tag's actual coordinate location.

\subsection{The result of master anchor optional algorithm}

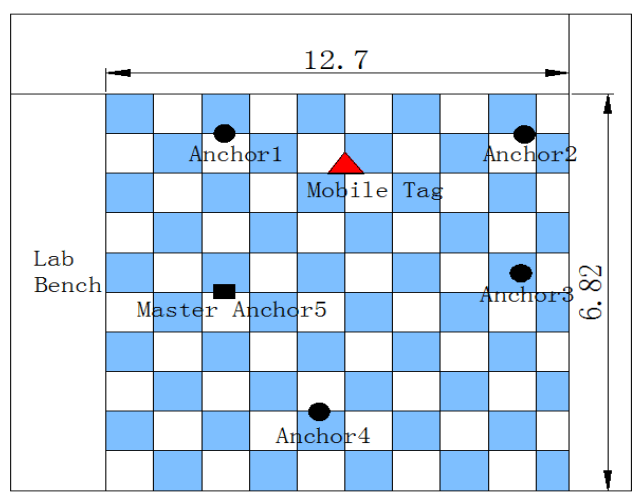

Figure 6. Experimental Environment

A lab room in Hainan University with the area $12.7 \mathrm{~m} \times 6.82 \mathrm{~m} \times 3 \mathrm{~m}$ served as experimental environment show in figure 6 .

They are five anchors in the space and only tag in our experiment. All the anchors are fixed and their location information will be sent to the server. In the experiment, all the anchors have two modes that is master anchor mode and slave anchor mode. For each location tested 50 times per second and tested 10 locations. First, we tested 10 locations when the master anchor works normal, the server will remember the distance between the tag and the anchor and then calculate the localization error which showed in figure 7 as line A. Line B represents the localization error when we put the master anchor in power off. The smallest localization error is $15.127 \mathrm{~cm}$ and the worst error is $24.901 \mathrm{~cm}$, as was shown by the test results.

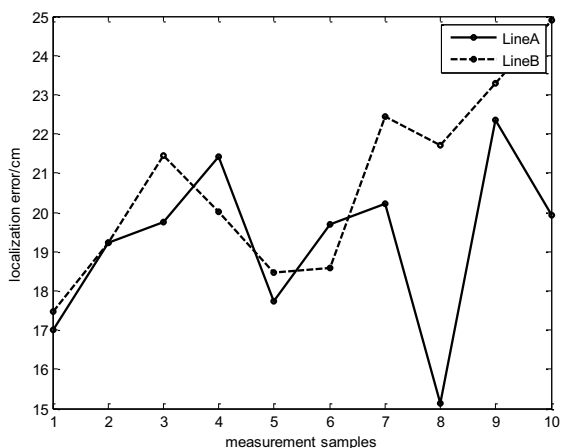

Figure 7. Localization error for master anchor optional algorithm

\subsection{The result of Network Scalability}

We did the experiment in a room with the area $26.7 \mathrm{~m} \times 8.24 \mathrm{~m} \times 3 \mathrm{~m}$. We used 8 anchors in our experiment. Two coord inate axes are established, and the anchor coordinates are fixed. Each anchor also has two modes and we tested 20 positions. Figure 8 is the histogram of statistical 50 times for one position. We set two master anchors at first and they are responsible for sending the clock synchronization information to the corresponding slave anchors. 


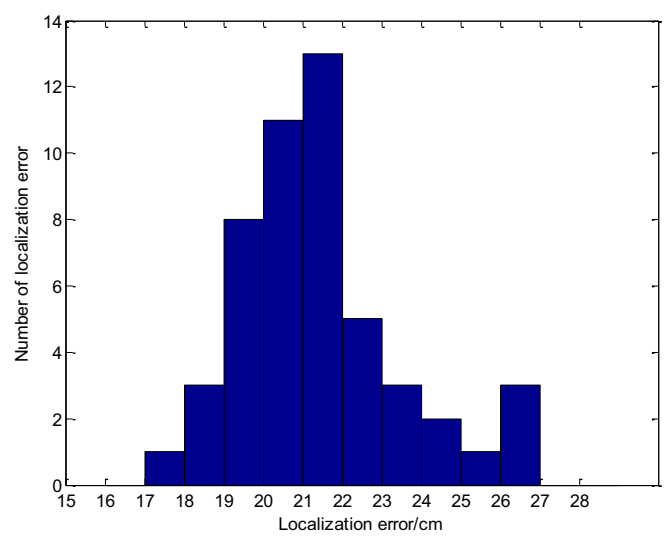

Figure8. Histogram of number of localization errors for one position

Figure 9 is the localization error about 20 measurement samples. From the figure, we can see that the positioning accuracy keeps in $15-28 \mathrm{~cm}$.

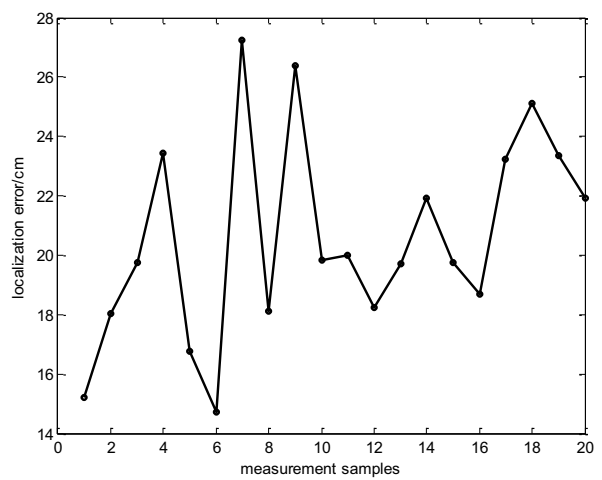

Figure 9. Localization error for network scalability

From the experiment we can see while the master anchor we first set was closed, the UWB indoor positioning system can automatically select the next anchor as a master anchor and in the experiment, the smallest localization error is $15.706 \mathrm{~cm}$ and the worst error is $27.230 \mathrm{~cm}$, as was shown by the test results.

\section{Conclusions}

In UWB indoor localization system, anchor clock synchronization error is the main performance limiting factor. In th is work, details of the theory on TDOA were given. UWB indoor positioning network adaptive algorithm which includes network scalability and the master anchor optional algorith $\mathrm{m}$ were used in experiment. The master anchor optional introduced that all the anchors have two modes. The Principle of Network Scalability introduced that when tag in a big room, all the anchors will be divided two regions and will keep relative clock synchronization.

In this study, we do two experiments in different room. Experimental results showed that the method can realize the scalability of adaptive network, ensure the positioning precision and enhance the communication ability of the network. In the experiment, the smallest localization error is around $15 \mathrm{~cm}$ and the worst error is around $28 \mathrm{~cm}$, as was shown by the test results. In future study we will combination GPS and UWB used in location of parking lot in the indoor and outdoor. And multi label positioning will be used in future studies and a larger improvement in the accuracy additional algorithms will be used in future studies.

\section{Acknowledgment}

This work was supported by Hainan EVK. The authors gratefully acknowledge the Hainan EVK and the financial support from China Natural Science Fund (Project No.61461017).

\section{References}

1. Wang T, Chen X, Ge N, and Pei, Y, "Error analysis and experimental study on indoor UWB TDoA localization with reference tag," IEEE Communications (APCC), 2013 19th Asia-Pacific Conference, 2013, pp. 505 - 508.

2. Choliz J, Equizabal M, Hernandez-Solana A, and Valdovinos, A, "Comparison of Algorithms for UWB Indoor Location and Tracking Svstems." IEEE Vehicular Technology Conference (VTC Spring), 2011 IEEE 73rd, pp. $1-5$.

3. Ghannouchi F M, Wang D, Tiwari S, "Accurate Wireless Indoor Position Estimation by Using Hybrid TDOA/RSS Algorithm," IEEE International Conference on Vehicular Electronics \& Safety (ICVES), 2012, pp.437-441.

4. He J, Li S, Pahlavan K, and Wang, Q, "A realtime testbed for performance evaluation of indoor TOA location system," IEEE International Conference on Communications, 2012, pp.482 - 486.

5. Dong Z, Wu Y, Sun D, "Data Fusion of the Real Time Positioning System Based on RSSI and TOF," IEEE International Conference on Intelligent Human-machine Svstems \& Cvbernetics. 2013. pp.503 - 506.

6. Hara, S., Anzai, D., Yabu, T., Lee, K., Derham, T., \& Zemek, R. (2013). A perturbation analysis on the performance of TOA and TDOA localization in mixed LOS/NLOS environments. Communications, IEEE Transactions on, 61(2), pp.679-689.

7. Kaune R, "Accuracy studies for TDOA and TOA localization," International Conference on Information Fusion. IEEE, 2012, pp.408-415.

8. D'Amico. A.. Taponecco. L.. and Mengali. U. (2013). Ultrawideband TOA estimation in the presence of clock frequency offset. Wireless Communications, IEEE Transactions on, 12(4), pp.1606-1616.

9. Sharp, I., Yu, K., \& Guo, Y. J. (2009). GDOP analysis for positioning svstem design. Vehicular Technology, IEEE Transactions on, 58(7), pp.3371-3382.

10. Shen, Y., \& Win, M.Z. (2010). Fundamental limits of Wideband localization-Part I: A general framework. Information Theory, IEEE Transactions on, 56(10), pp. 4956-4980.

11. Abbasi A, Kahaei, M H, "Improving source localization in LOS and NLOS multipath environments for UWB signals,". In Computer Conference, 2009. CSICC 2009. 14th International CSI. IEEE, 2009, pp.310-316.

12. Lei. Y. P.. Gong. F. X.. \& Ma. Y. O. "Optimal distribution for four-station TDOA location system," In Biomedical Engineering and Informatics (BMEI), 2010 3rd International Conference on (Vol. 7). IEEE. 2010, October, pp. 2858-2862. 